\title{
Growth and expression of rat bone marrow mesenchymal stem cells modified by nerve growth factor in diabetic rat bladders
}

\author{
SHAO-XING ZHU ${ }^{1}$, SHI-YONG HUANG ${ }^{2}$, YI-MING SU ${ }^{3}$ and PENG CAI ${ }^{4}$ \\ ${ }^{1}$ Department of Urology, Zhejiang Cancer Hospital, Hangzhou 310022; ${ }^{2}$ Department of Urology, \\ Xiamen Hospital of Traditional Chinese Medicine, Xiamen 361000; ${ }^{3}$ Department of Urology, Union Hospital, \\ Fujian Medical University, Fuzhou 350001; ${ }^{4}$ Department of Urology, Xiangcheng Hospital, Zhangzhou 363000, P.R. China
}

Received November 15, 2012; Accepted March 13, 2013

DOI: $10.3892 / \mathrm{mmr} .2013 .1425$

\begin{abstract}
The aim of the present study was to determine whether rat bone marrow mesenchymal stem cells (MSCs) transfected with the nerve growth factor (NGF) gene and then transplanted into diabetic rat bladder tissues survive and continue to express NGF. A recombinant lentiviral vector carrying the NGF gene was constructed and transfected into rat bone marrow MSCs. BrdU-labeled immunohistochemistry was used to observe NGF expression in the transfected MSCs. BrdU-labeled and NGF-transfected MSCs were transplanted into diabetic rat bladder tissues. BrdU-labeled immunohistochemistry was used to observe the growth of NGF-transfected MSCs in the tissue samples. NGF mRNA and protein expression levels in MSCs were analyzed using reverse transcription polymerase chain reaction (RT-PCR) and ELISA, respectively. The recombinant NGF gene lentiviral vector and NGF gene-modified rat bone marrow MSCs were successfully constructed. NGF gene-modified rat MSCs survived in the diabetic rat bladders 4 weeks following injection and NGF gene expression was increased. In the present study, NGF gene-modified MSCs were shown to be capable of survival in diabetic rat bladder tissues and stably expressed NGF.
\end{abstract}

\section{Introduction}

Diabetes is a chronic disease that poses a serious threat to human health. Diabetic patients $(\sim 85 \%)$ commonly have concurrent bladder dysfunction symptoms, which are often referred to as diabetic cystopathy. In general, patients lose bladder-filling sensation and have decreased bladder contrac-

Correspondence to: Professor Shao-Xing Zhu, Department of Urology, Zhejiang Cancer Hospital, 38 Guangji Road, Gongshu, Hangzhou 310022, P.R. China

E-mail: sxcpcn@163.com

Key words: nerve growth factor, bone marrow mesenchymal stem cells, diabetic cystopathy, gene, lentiviral vector tion activity, which leads to increases in bladder capacity and late residual urine volume. In the late stages of this disease, urinary tract function is impaired, causing hydronephrosis and uremia (1). The pathogenesis of diabetic bladder dysfunction occurs via diabetes-induced damage to the main sensory and autonomic nerve system in the bladder (2).

In previous years, the correlation between nerve growth factor (NGF) and diabetes with neuropathy has caused widespread concern. Hellweg et al (3) reported that NGF is important for reducing the incidence of diabetic neuropathy from the sensory nerve pathways to the target organ axons antiporter. Sasaki et al (4) showed that NGF levels decreased with increasing time in the bladder and L6 S1 dorsal root ganglia and function of $\mathrm{A} \delta$ and $\mathrm{C}$ fibers in the afferent nerve was impaired, causing bladder voiding dysfunction. Preliminary results of the study revealed that exogenous NGF may represent an effective treatment for diabetic neuropathy. In 2001, Goins et al (5) reported the first use of defective recombinant herpes simplex virus (HSV) as a carrier of the NGF gene into the rat bladder wall and identified an increase in NGF levels in the bladder and dorsal root ganglion. In 2004, Sasaki et al (4) reported that HSV vector-mediated NGF treatment of the diabetic urinary bladder in animal studies confirmed that gene therapy significantly increased expression of NGF in the bladder and bladder afferent pathways and improved urinary bladder function (6).

Stem cells are a class of cells with self-renewal capacity and a high degree of proliferation and differentiation potential. Stem cell research in urology has gradually increased. Yokoyama et al (7) reported that skeletal muscle-derived stem cells injected into the bladder and urethra wall may be capable of long-term survival. Strasser et al (8) treated 20 stress urinary incontinence patients with autologous stem cell injections into skeletal muscle. Complete remission of urinary incontinence symptoms was reported in 18 patients. A bladder wall injection of stem cells improves bladder function by the differentiation of stem cells into smooth muscle cells and these cells may also function as vectors for gene therapy. Huard et al (9) incorporated the $\beta$-gal gene into skeletal muscle stem cells and injected these stem cells into the bladder wall. The authors reported that cells expressing this gene were able to differentiate into smooth muscle cells and improved contractility was observed in the damaged bladder. 
These studies indicate that transgenic technology coupled with cell engineering technology has potential application in the treatment of urinary system dysfunction.

Diabetic bladder dysfunction is an extremely difficult clinical issue and currently lacks effective treatment. Preliminary results have indicated that NGF gene transfer improves bladder voiding function. Since mesenchymal stem cells (MSCs) have multi-lineage differentiation potential to differentiate into smooth muscle and also act as a vehicle for gene therapy, a recombinant lentiviral NGF vector was constructed and transfected into MSCs to generate stable expression of NGF in the present study. NGF gene-modified MSCs were injected into bladder tissues and MSC survival rate and expression of the NGF gene were observed.

\section{Materials and methods}

Materials. An inbred strain of Sprague Dawley (SD) rats was purchased from Shanghai Silaike Experimental Animals Co., Ltd. (QZ:SCXK; Shanghai, China). This study was performed in strict accordance with the recommendations in the Guide for the Care and Use of Laboratory Animals of the National Institutes of Health. The animal use protocol was reviewed and approved by the Institutional Animal Care and Use Committee of Zhejiang Cancer Hospital.

Lentivirus for NGF recombination. A $\beta$-NGF sequence was amplified from pGC-E1-NGFB by PCR and cloned into the pGC-FU lentivirus vector to construct pGC-FU- $\beta$-NGF. The plasmid was confirmed by digestion and sequencing. pGC-FU- $\beta$-NGF and pHelper 1.0 and 2.0 were transfected into the human embryonic kidney cell line $293 \mathrm{~T}$ to obtain recombinant lentivirus GU-FU- $\beta$-NGF, which encodes the $\beta$-NGF gene.

Rat MSCs. Rat MSCs were separated, cultivated and identified by whole bone marrow adherent culture. The third generation of rat MSCs was separated to detect osteogenesis- and lipogenesis-induced differentiation by Alizarin red staining and Oil red $\mathrm{O}$ staining. The assay was performed using flow cytometry to detect surface CD markers of MSCs.

Lentivirus infection. Rat MSCs were infected with lentiviruses carrying the GU-FU- $\beta$-NGF and GFP vectors. GFP expression was observed by fluorescence microscopy. NGF mRNA was detected using real-time polymerase chain reaction (PCR), while NGF expression was examined by ELISA.

Diabetic rat model. SD rats (weight, 180-200 g) were selected and fasted for $12 \mathrm{~h}$. Rats were divided into the diabetic and control groups. The diabetic group was treated with intraperitoneal injections of $60 \mathrm{mg} / \mathrm{kg} /$ time STZ, while the control group was treated with citric acid buffer at the same volume. Venous blood glucose (BG) was analyzed $72 \mathrm{~h}$ following injection. $\mathrm{BG}$ level $>16.7 \mathrm{mmol} / \mathrm{l}$ was considered to indicate the successful induction of diabetes.

BrdU-labeled rat MSCs. BrdU (1 g) was dissolved in $100 \mathrm{ml}$ citric acid buffer. NGF-transfected rat MSCs were digested with trypsin and plated onto pre-treated 6-well plates at a density of $1 \times 10^{5}$ cells $/ \mathrm{ml}$. MSCs were incubated with $10 \mu \mathrm{mol} / 1$ BrdU for $40 \mathrm{~h}$ when cell density reached $40 \%$.

BrdU-labeled diabetic rat bladders. BrdU was incubated with NGF-transfected rat MSCs for $48 \mathrm{~h}$. Cells were digested with trypsin and centrifuged at $157 \mathrm{x}$ g for $5 \mathrm{~min}$. Cells were suspended in phosphate-buffered saline (PBS) to a concentration of $2 \times 10^{5}$ cells $/ \mathrm{ml}$. Rats were anesthesized using intraperitoneal injections of $10 \%$ chloral hydrate $(0.3 \mathrm{ml} / 100 \mathrm{~g}$ body weight). Following, disinfection, a vertical incision $(\sim 1.0 \mathrm{~cm})$ was made in the rat abdomen and the bladder was exposed. Suspended cells $(10 \mu \mathrm{l})$ were injected into the bladder muscle using a microsyringe; a total of 10-20 points on the muscle were injected. The incision was closed and the rats were revived.

Immunohistochemistry. Rat bladders were removed 4 weeks following transplantation of NGF-transfected MSCs labeled by BrdU. Samples were fixed with $10 \%$ formaldehyde, immersed, flooded, sliced, spread, dried at $75^{\circ} \mathrm{C}$ for $1 \mathrm{~h}$ and waxed. The samples were then incubated with $3 \% \mathrm{H}_{2} \mathrm{O}_{2}$ for $20 \mathrm{~min}$ to block endogenous catalase and antigens were repaired by microwave. The samples were incubated with $0.5 \%$ Triton X-100 and washed with PBS. Next, the samples were incubated with $1 \%$ bovine serum albumin for $10 \mathrm{~min}$ to block non-specific antigens following $2 \mathrm{~N} \mathrm{HCl}$ incubation for $30 \mathrm{~min}$. The samples were incubated with a monoclonal BrdU antibody $(1: 1,000)$ at $37^{\circ} \mathrm{C}$ for $2 \mathrm{~h}$. Samples were incubated with reagent 1 at $37^{\circ} \mathrm{C}$ for $2 \mathrm{~h}$ and then reagent 2 for $2 \mathrm{~h}$. Next, the samples were developed with 3,3'-diaminobenzidine and stained with hematoxylin for $30 \mathrm{sec}$. Observations were performed by microscopy. Rat bladder MSC samples without BrdU incubation were set as the negative control group.

Reverse transcription RT-PCR. RNA was extracted from rat bladder using TRIzol. Reverse transcription and PCR were performed according to the manufacturer's instructions. Primer sequences were as follows: NGF, upstream AACAGGACTCACAGGAGCAA and downstream CTTCCTGCTGAGCACACACA (369 bp); and GAPDH (control), upstream CAAGGTCATCCATGACAACTTTG and downstream GTCCACCACCCTGTTGCTGTAG (496 bp). The annealing temperature was set at $58^{\circ} \mathrm{C}$ and the reaction was repeated for 35 cycles. PCR products were confirmed by $2 \%$ agarose gel electrophoresis. Gel images were captured using the Quantity One image analysis system. Expression of $\mathrm{NGF} / \mathrm{GAPDH}$ was scored by the grayscale value of each band. NGF gene expression was determined by the ratio of NGF and GADPH expression.

ELISA assay. Rat bladders were removed 8 weeks following MSC transplantation. Experimental subgroups were the control (normal rat bladder), diabetic (diabetic rat bladder) and treatment groups (diabetic rat bladder transplanted with MSCs transfected with the NGF gene). Rat bladder tissue samples were thawed gradually and dried following rinsing with cold saline. The bladders were weighed and placed into 10 times volume saline. The samples were sheared, homogenized with a tissue homogenizer and centrifuged for $12 \mathrm{~min}$. Supernatant was assayed using an ELISA kit according to the manufacturer's instructions. 

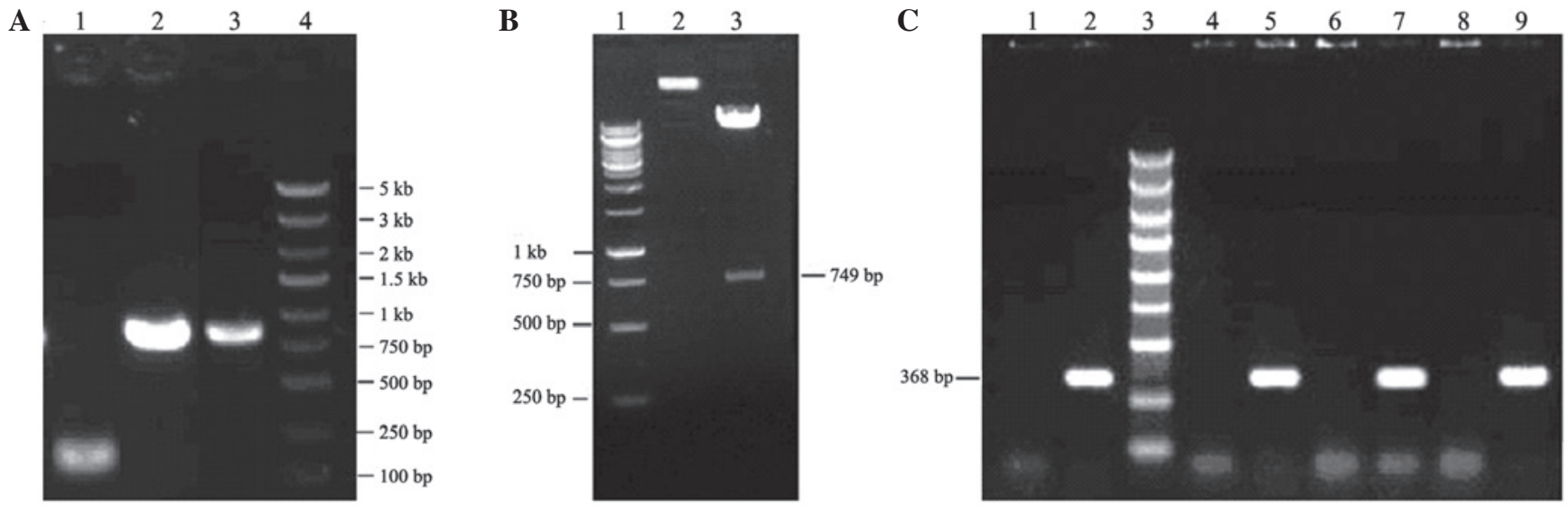

D Chromas 1.45 File: A02_B0619288.164-1.PGC-E-SEQ Sequence Name: A02_B0619288.164-1.PGC-E-SEQR Run ended: Jun 21, $2007 \quad$ Page 1 of 2
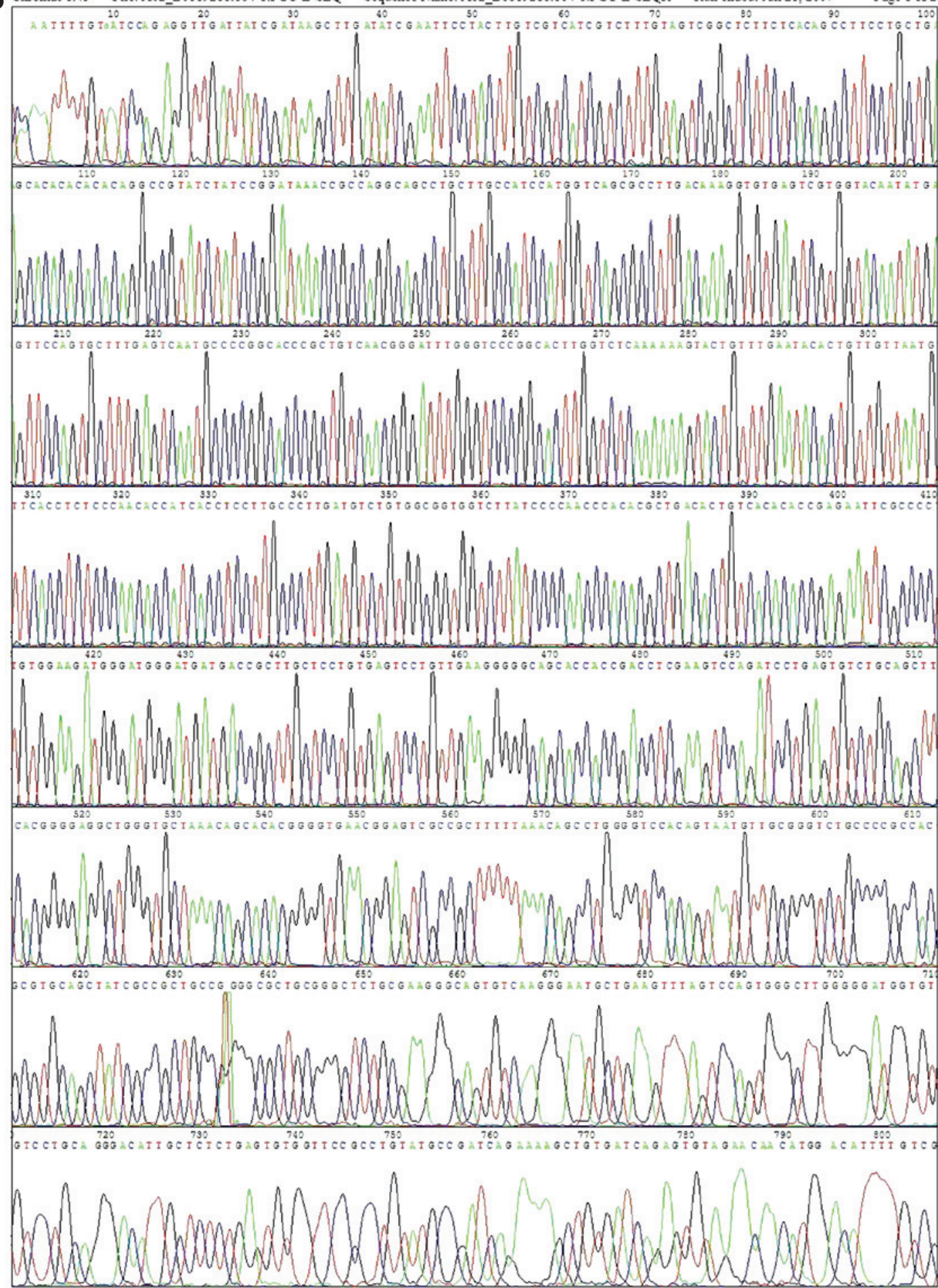
$\mathbf{E}$

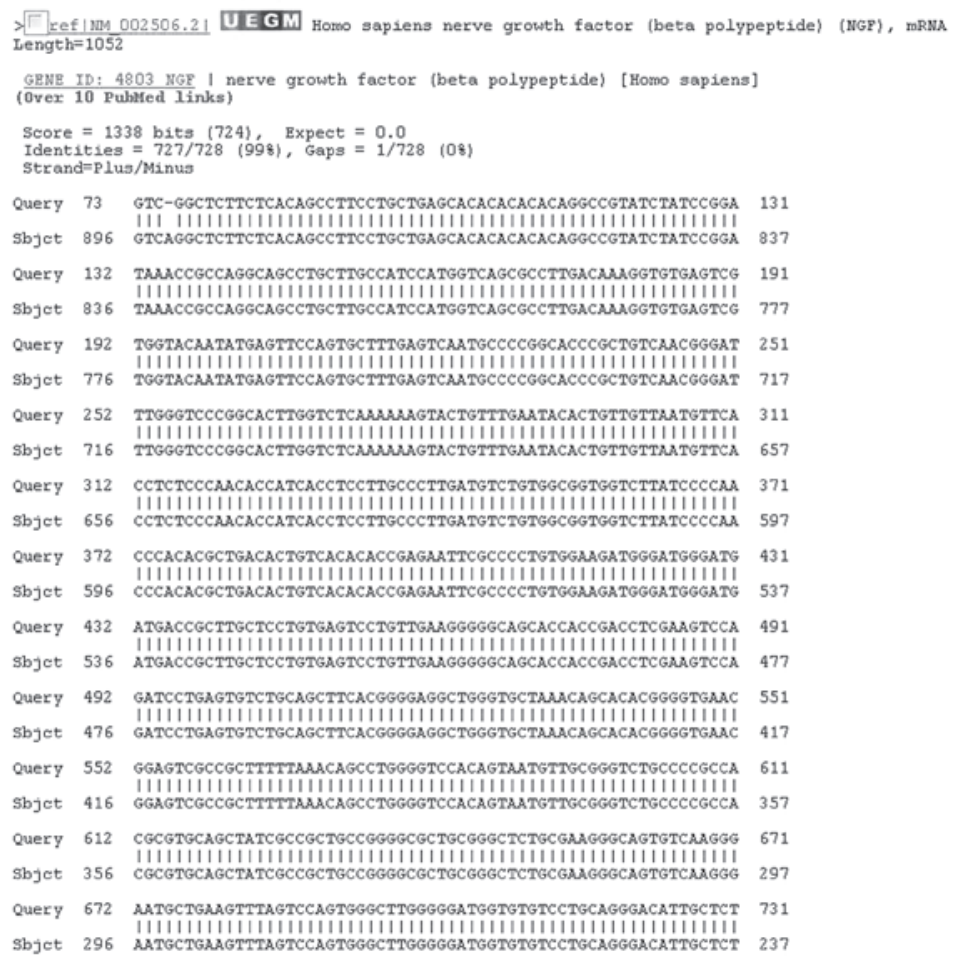

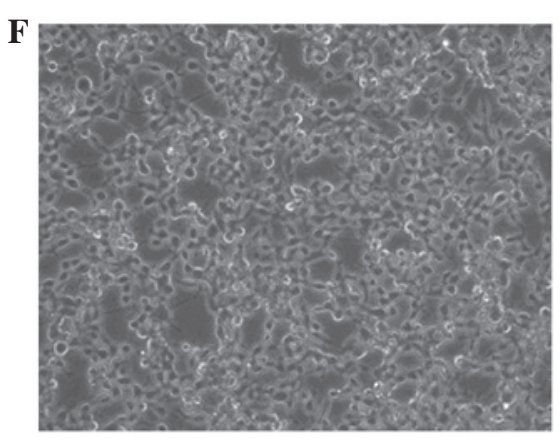

Statistical analysis. Data are presented as the mean \pm SD and were analyzed using SPSS 13.0. Data associated with NGF lentivirus transfection and diabetic rat modeling were analyzed by a t-test (independent samples test). ELISA and RT-PCR results were analyzed by one-way ANOVA. Comparison analysis between groups was performed with Student-Newman-Keuls law. $\mathrm{P}<0.05$ was considered to indicate a statistically significant difference.

\section{Results}

Lentivirus preparation. Agarose gel electrophoresis of PCR products is presented in Fig. 1A, which demonstrates that 3FLAG and $\beta$-BGF genes were successfully amplified. PCR product bands were of the expected sizes; 102, 792 and $872 \mathrm{bp}$. Agarose gel electrophoresis of the double digested products of lentivirus vector pGC-FU are demonstrated in Fig. 1B. The digestion was successful since there were 2 bands of $\sim 10$ and $749 \mathrm{~kb}$, which were the expected product sizes.

Confirmation of recombination of pGC-FU- $\beta$-NGF by PCR is revealed in Fig. 1C. Bands at 368 bp in lanes 5, 7 and 9 were consistent with predicted PCR products. Therefore, the plasmid recombination was considered to be successful.
Figure 1.Preparation of lentivirus for NGF reorganization. (A) Electrophoresis of PCR products: Lanes 1, 3FLAG; $2, \beta$-NGF; 3, 3FLAG + $\beta$-NGF; and 4 , marker. 3FLAG and $\beta$-BGF genes were successfully amplified. PCR product bands were of the expected sizes; 102, 792 and 872 bp. (B) pGC-FU was identified by double digestion: Lanes $1,1 \mathrm{~kb}$ marker; 2 , pGC-FU without digestion; and 3, pGC-FU digested with Age I and EcoR I. (C) pGC-FU- $\beta$-NGF was identified by PCR: Lanes 1 , negative control (vector); 2, positive control (vector inserted with fragments which had been sequence-identified); 3, marker; and 4-9, PCR products of recombination plasmid. (D) Sequencing of the positive clone; (E) BLAST analysis between sequence of the positive clone and standard sequence. (F) Co-transfection of 293 T cells with three plasmids (bright field; magnification, x100). NGF, nerve growth factor.

The positive plasmid was purified and sequenced. BLAST analysis between the plasmid and standard sequences was consistent, which further confirmed plasmid recombination (Fig. 1D). BLAST analysis between sequences of the positive clone and standard is presented in Fig. 1E. Query represents the positive clone plasmid sequence and subject represents the standard sequence from GenBank. The identity of BLAST analysis was 99\% (727/728), while the missing base revealed in BLAST does not exist. Since the coding DNA sequence of $\beta$-NGF is located between 170 and $895 \mathrm{bp}$, the location revealed in BLAST represents a termination codon (UGA). Therefore, the sequence for the recombinant plasmid was correct. Fig. 1F demonstrates lentiviral packaging by co-transfection to $293 \mathrm{~T}$ cells with three plasmids.

Cell culture and identification. Cells were round up when first inoculated into culture flasks, while the majority of cells were suspended in cell culture medium. The majority of cells were attached to the surface after $48 \mathrm{~h}$ and unattached cells were removed. The morphologies of attached cells were not homogeneous, exhibiting fusiform fibroblastic or polygonal appearances. The cell nucleus was larger and located at the center or edge of the cell. Dispersive attached fibroblasts 

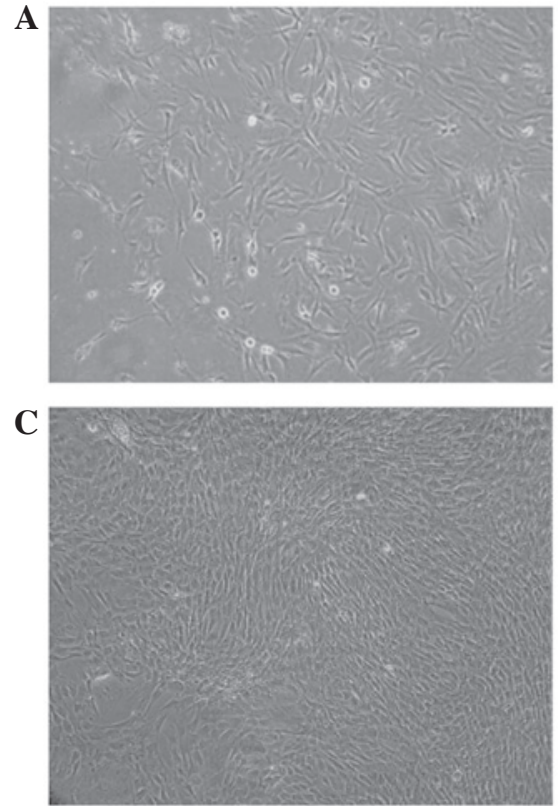

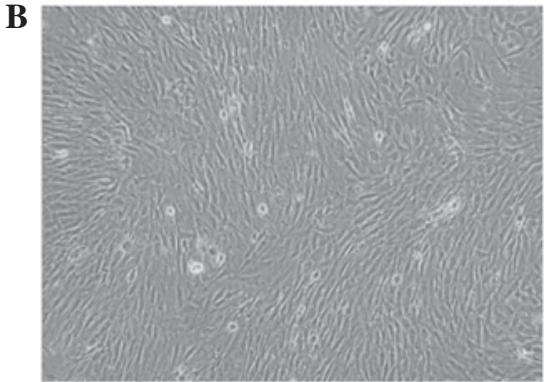

D

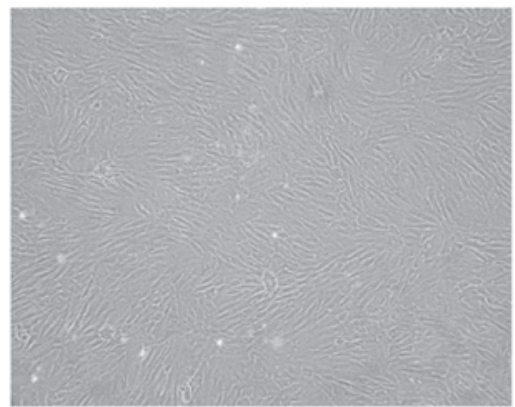

Figure 2. Morphological observations of rat bone marrow. Primary culture at (A) day 5 and (B) day 9; (C) day 4 of passage 2 MSCs; and (D) day 4 of passage 5 MSCs. MSCs, mesenchymal stem cells.

appeared at days 3 and 4 and cells formed colonies following days 5 and 6; the colonies proliferated rapidly (Fig. 2A). Each colony was formed of 100-200 cells (Fig. 2D). Cells demonstrated a spindle fibroblastic appearance, while certain cells exhibited a broad and flat polygonal appearance. After 9-11 days, cells formed a monolayer indicating $>90 \%$ fusion and the vertical axis of spindle cells were arranged parallel to each other (Fig. 2B).

Cells were completely attached by $24 \mathrm{~h}$ and reached complete fusion in 3-4 days, exhibiting a unique spindle appearance (Fig. 2C and D). After 10 generations, cells maintained vigor in growth and expansion. No significant change in fusion time was identified.

Expression of surface markers, CD90, CD44 and CD106, was detected with flow cytometry. The expression levels of CD90, CD44 and CD106 were 91.50, 99.87 and 78.02\%, respectively. CD34, CD45 and CD11 expression levels were low, at 0.07, 0.15 and $0.21 \%$, respectively (Fig. 3).

MSCs were stained with alizarin red following 21 days of induction. The induced group revealed a large number of cells with an orange mineralization nodal morphology, while control cells were negative for these properties (Fig. 4A and B).

Oil red $\mathrm{O}$ staining of MSCs following 2 weeks of induction demonstrated numerous intracellular lipid droplets around the nucleus and lined up in bright columns, while the cells revealed various long spindle appearances. Partial lipid droplets merged into larger conglomerations and moved the nucleus aside. A low number of red lipid droplets were observed in the control group (Fig. 4C and D).

Recombinant NGF lentivirus infection of rat MSCs. Rat MSCs infected with the lentiviral GFP-tagged vector for $48 \mathrm{~h}$ revealed green fluorescence. The infection rate was estimated to be $>95 \%$ by comparing green and bright field cells (Fig. 5A and B). Green fluorescence was observed in whole cells and was sustainably expressed through generations, indicative of successful GFP vector transfection. Since the transfection conditions of the GFP vector and recombinant NGF were the same, these observations indicated that the NGF gene was successfully transfected into MSCs.

Reverse transcription RT PCR augmentation curves (Fig. 5E) demonstrated that GAPDH, which was set as a PCR control, was expressed at similar levels in the experimental and control groups. Threshold cycle $(\mathrm{Ct})_{\mathrm{GADPH}}$ values in the two groups were within the same range (Table I). Statistical analysis revealed that there was no significant difference in GAPDH expression between the groups.

By contrast, a significant difference in NGF expression was identified between the two groups. $\mathrm{Ct}_{\mathrm{NGF}}$ was $>33$ in the control group and there were miscellaneous peaks in the gene melting curve (Fig. 5G), indicating that the NGF gene was not expressed in the control group. In addition, $\mathrm{Ct}_{\mathrm{NGF}}$ was 18.46 in the experimental group, which was revealed to be significantly different from the control $(\mathrm{P}<0.05)$. The melting curves were normal (Fig. 5F and G). Therefore, the expression of NGF was higher in the NGF lentivirus-infected group compared with the control group. Using the $2^{-(\Delta \Delta C T)}$ method, relative expression of the NGF gene was calculated as $2^{13.73}=13,587.57$.

The MSCs were cultured for 48-72 h following infection. Supernatants were obtained from the experimental and control groups. An ELISA assay was performed as described. NGF levels in the experimental and control groups were $190 \pm 41.34$ and $0 \mathrm{pg} / \mathrm{ml}$, respectively, as NGF protein was not detected in the control group $(\mathrm{P}<0.01$; Fig. $5 \mathrm{H})$.

Diabetic rat modeling. Blood sugar levels of rats increased $72 \mathrm{~h}$ following intraperitoneal injection with STZ. Levels remained high 8 weeks later in diabetic rats $(n=15)$ compared with control rats $(n=15)$. Compared with the controls, the diabetic group was shown to have higher blood sugar levels $(\mathrm{P}<0.05)$, decreased weight $(\mathrm{P}<0.05)$, increased urine volume $(\mathrm{P}<0.05)$ and increased bladder wet weight $(\mathrm{P}<0.05)$. In addition, the fur 
A

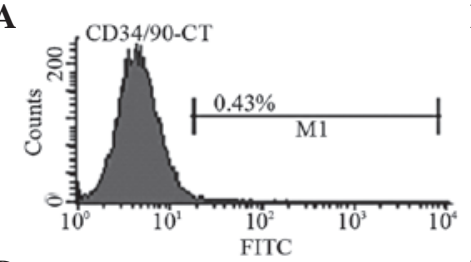

D

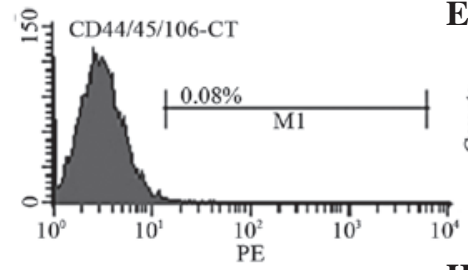

G

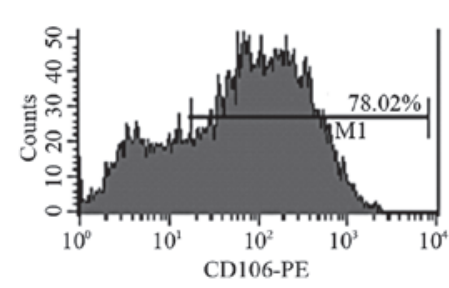

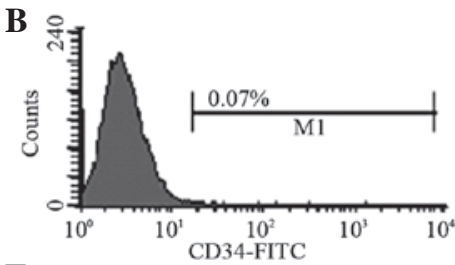

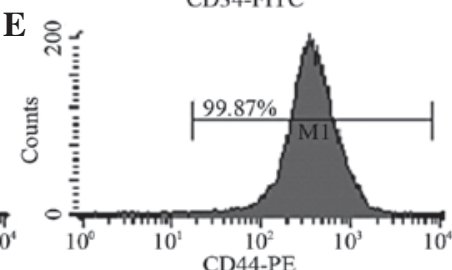

$\mathbf{H}$

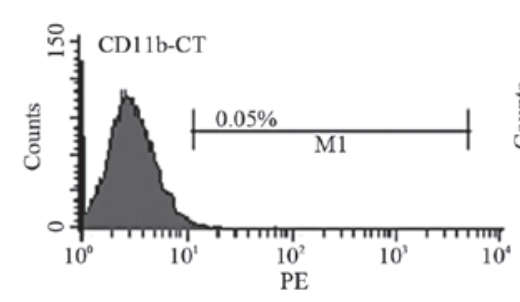

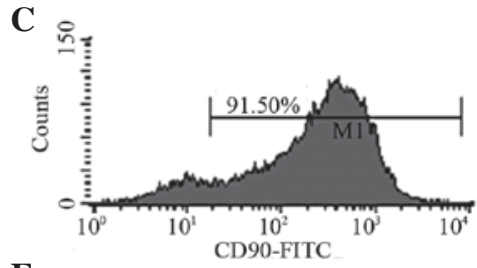

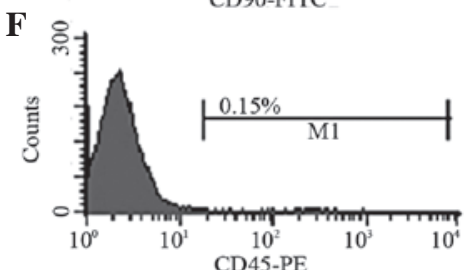

I

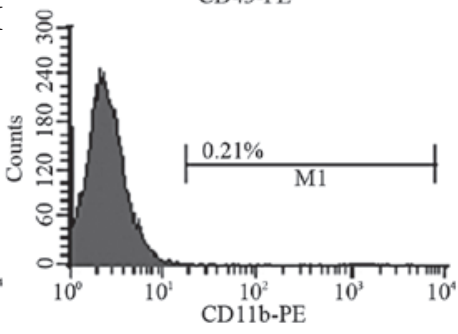

Figure 3. Flow cytometry of surface marker expression of passage 3 rat bone marrow MSCs. (A) Fluorescein isothiocyanate (FITC)-isotypism and (D and H) PE-isotypism controls. Expression of (B) CD34, (C) CD90, (E) CD44, (F) CD45, (G) CD106 and (I) CD11b. MSCs, mesenchymal stem cells.
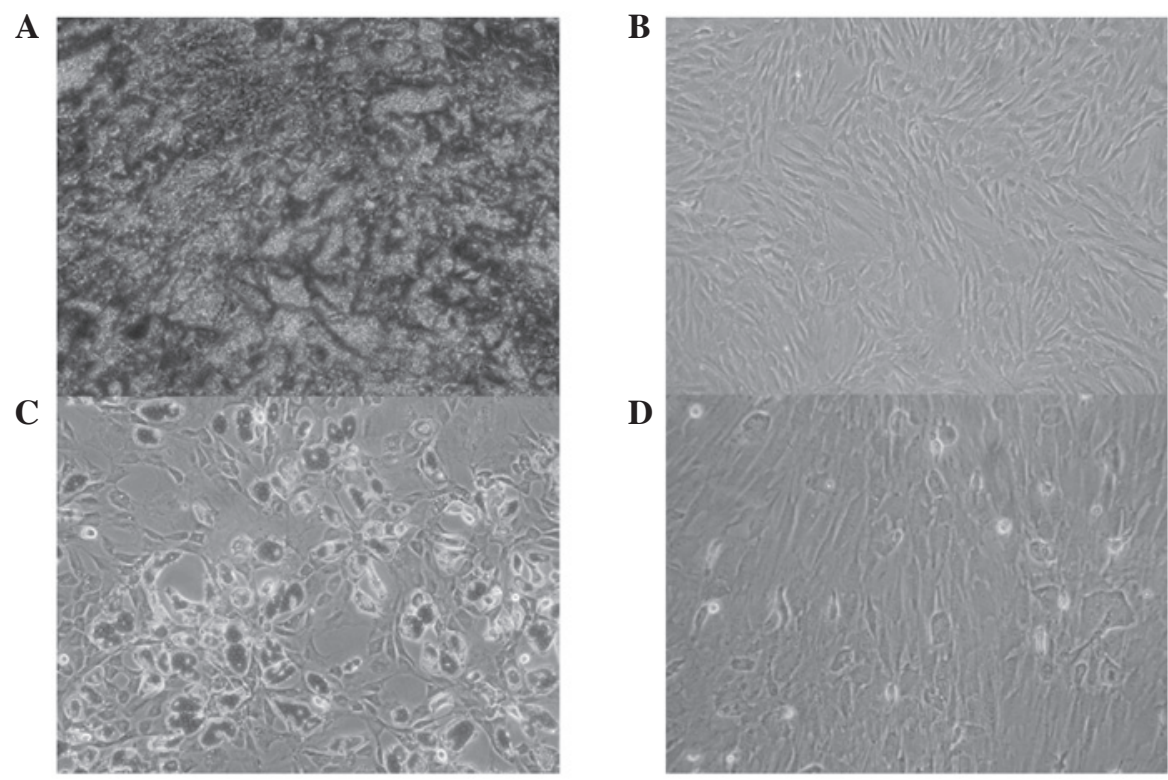

Figure 4. Identification of induction of osteogenic and adipogenic differentiation. (A and B) Alizarin red staining following induction of bone marrow mesenchymal stem cell differentiation into osteoblasts (magnification, x100); (A) experimental and (B) control groups; (C and D) Oil Red O staining following induction of bone marrow mesenchymal stem cell differentiation into adipocytes (magnification, x200); (C) experimental and (D) control groups.

of diabetic rats lost quality, while drinking water volume and food intake increased. These observations indicate that the diabetic rat model was established (Table II).

BrdU-labeled NGF transfected MSCs. NGF-transfected rat MSCs were incubated with $10 \mu \mathrm{M} \mathrm{BrdU}$ for $48 \mathrm{~h}$. The cell nucleus was stained brown by immunohistochemistry and staining efficiency was $>80 \%$. The nuclear negative control was stained blue (Fig. 6A and B).
Immunohistochemistry. Detection of BrdU-labeled MSCs was performed 4 weeks following transplantation and indicated that the MSCs had survived in the rat bladder. Sporadic brown markings could be observed in bladder muscle cells, while positive staining was not identified in the control group (Fig. 6C and D).

Reverse transcription RT-PCR. NGF and GAPDH mRNA levels of the control, diabetic and treatment groups were 
A

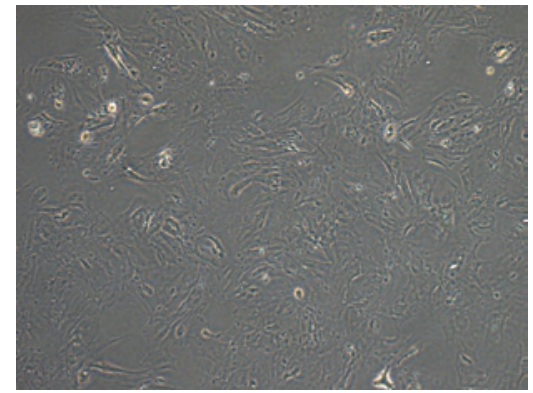

C

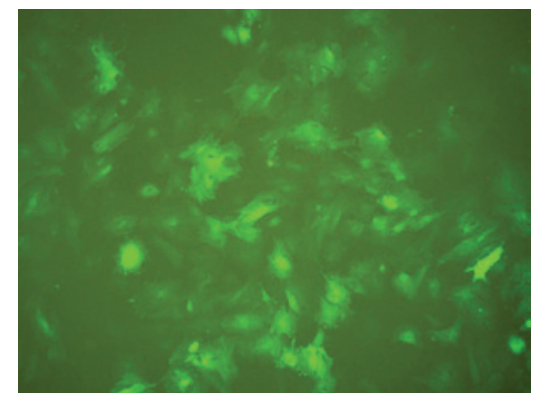

$\mathbf{E}$

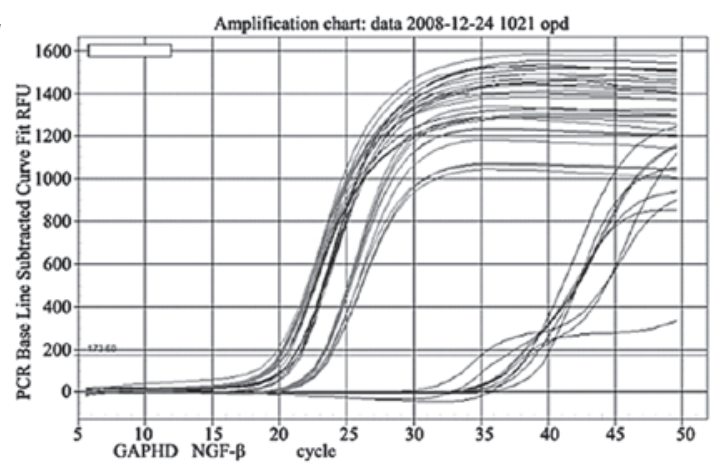

G

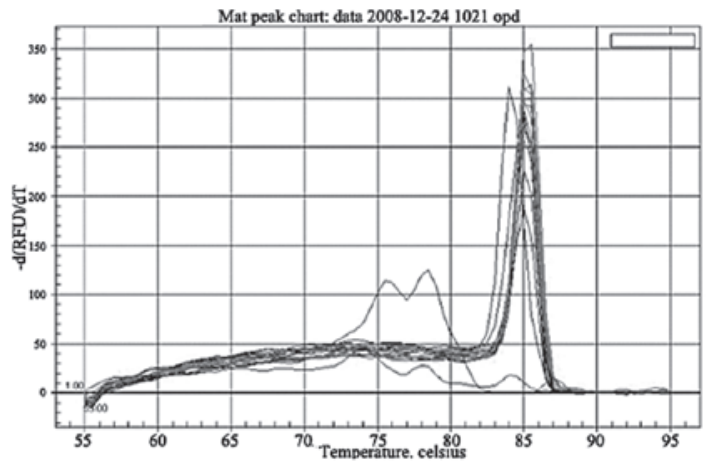

B

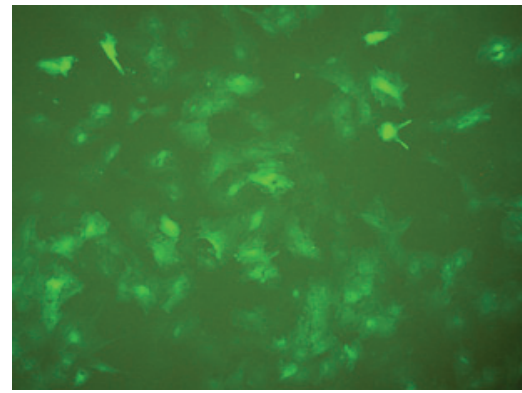

D

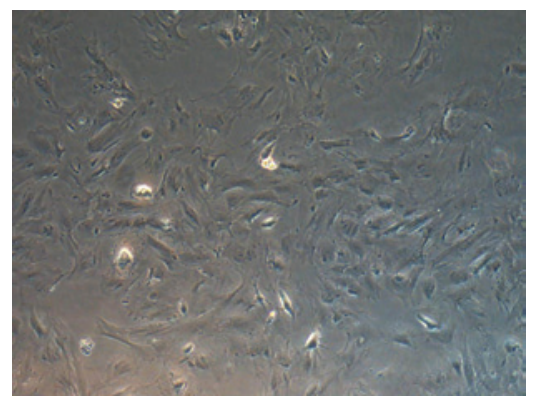

$\mathbf{F}$

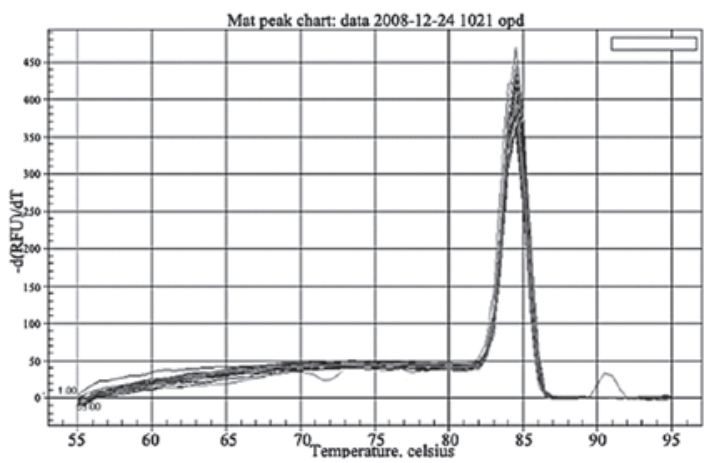

H

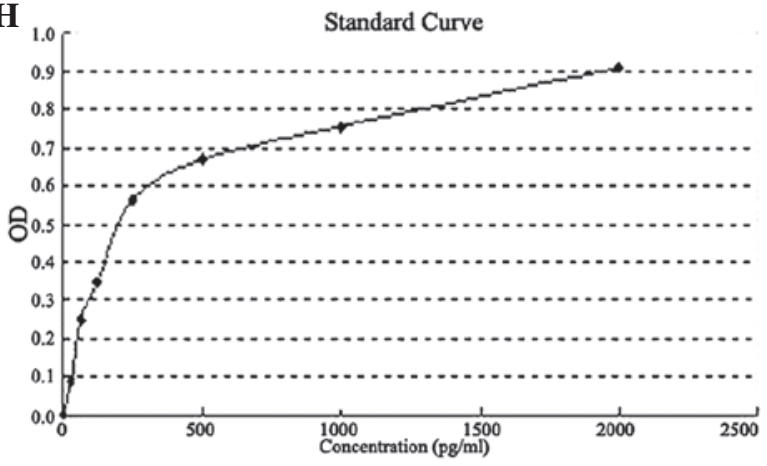

Figure 5. Gene expression levels of NGF in rat MSCs. (A-D) Expression of green fluorescence in rat MSCs infected with lentiviral vector containing GFP (magnification, x100); (A and B) represent the same field of vision, demonstrating that rat MSCs were infected with the lentiviral vector after 48h. (C and D) represent the same field of vision, revealing that rat MSCs were infected with the lentiviral vector 3 days following cell passage. (A and D) bright and (B and C) fluorescent fields. qPCR analysis of NGF and GAPDH (housekeeping) gene expression. (E) Amplification curves of GAPDH and NGF genes. Melting curves of (F) GAPDH and (G) NGF genes. (H) Standard curve of NGF by ELISA detection. PCR, polymerase chain reaction; RFU, relevant fluorescent units; OD, optical density; NGF, nerve growth factor; MSCs, mesenchymal stem cells.

analyzed by reverse transcription RT-PCR (Fig. 6E). NGF mRNA levels in the diabetic group were lower compared with the control group $(\mathrm{P}<0.05)$. Analysis was performed 4 weeks following MSC transplantation, indicating that NGF expression was greater in the treatment compared with the diabetic group $(\mathrm{P}<0.05$; Table III).

ELISA. An ELISA assay was performed using supernatants obtained from each group. NGF protein expression in the control, diabetic and treatment groups were 113.837 \pm 3.044 ,
$70.057 \pm 1.953$ and $109.943 \pm 2.347 \mathrm{pg} / \mathrm{ml}$, respectively. The expression of NGF was reduced in the diabetic compared with the control group $(\mathrm{P}<0.05)$. NGF protein levels in the treatment group recovered to control levels (Table III).

\section{Discussion}

Use of the correct vector is an important factor for stable gene expression in gene therapy. A previous study demonstrated low levels of transfection efficiency using plasmids, 

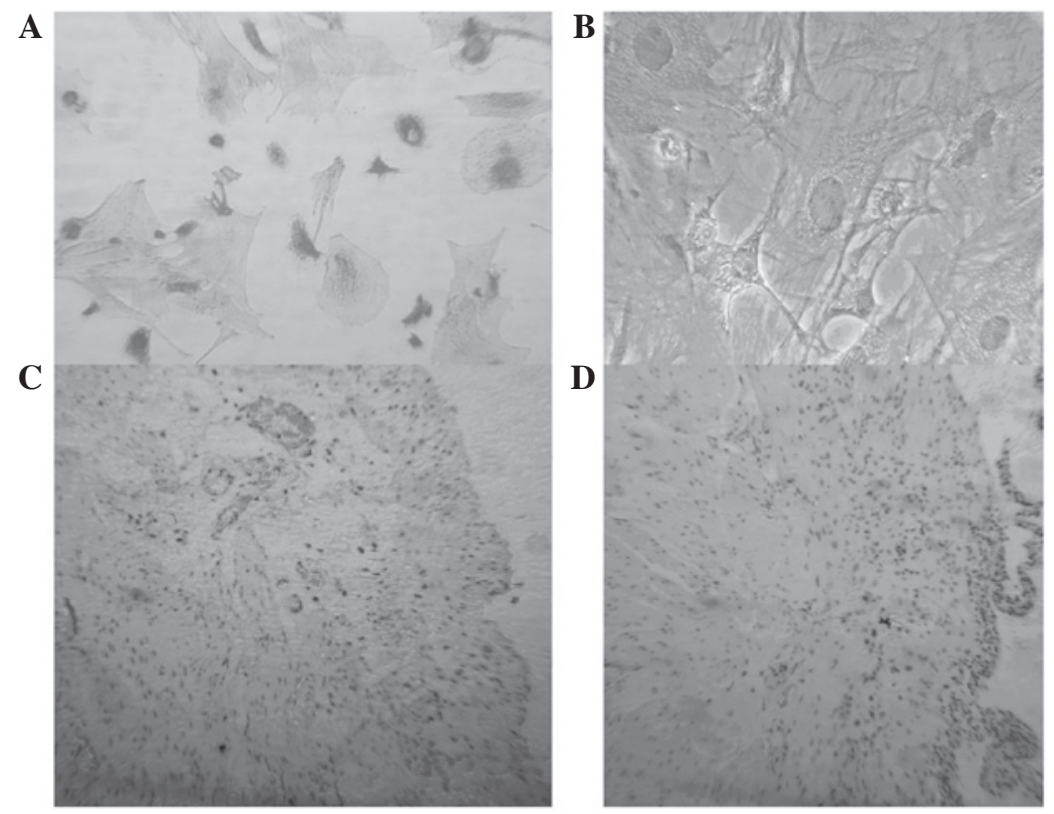

$\mathbf{E}$

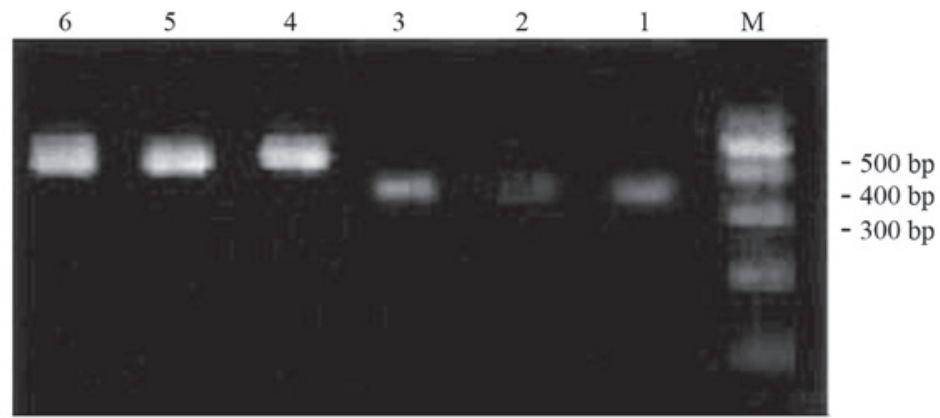

Figure 6. Growth of implanted rat MSCs and gene expression of NGF in diabetic rat bladder tissue. BrdU labeled MSCs; (A) experimental and (B) negative control groups (magnification, $\mathrm{x} 400$ ). BrdU labeled MSCs in rat bladder; (C) experimental and (D) negative control groups (magnification, x100). (E) RT-PCR and electrophoresis of the control, diseased and treatment groups. MSCs, mesenchymal stem cells; NFG, nerve growth factor; RT-PCR, reverse transcription polymerase chain reaction.

Table I. Ct values of rat MSCs transfected with NGF (experimental group) or empty vectors (control group).

\begin{tabular}{lcc}
\hline Group & $\mathrm{Ct}_{\mathrm{GAPDH}}$ & $\mathrm{Ct}_{\mathrm{NGF}}$ \\
\hline Control & $16.02 \pm 0.46$ & $33.02 \pm 1.40$ \\
Experimental & $15.19 \pm 0.36^{\mathrm{a}}$ & $18.46 \pm 0.30^{\mathrm{b}}$ \\
\hline
\end{tabular}

${ }^{\mathrm{a} P}>0.05$ vs. control, ${ }^{\mathrm{b}} \mathrm{P}<0.05$ vs. control. MSCs, mesenchymal stem cells; NGF, nerve growth factor. $\mathrm{Ct}$, threshold cycle.

Table II. Blood glucose, body weight, urine volume and bladder wet weight in the diabetic and control groups.

\begin{tabular}{lcc}
\hline Characteristics & $\begin{array}{c}\text { Diabetic } \\
\text { group }(\mathrm{n}=15)\end{array}$ & $\begin{array}{c}\text { Control } \\
\text { group }(\mathrm{n}=15)\end{array}$ \\
\hline Blood glucose $(\mathrm{mmol} / \mathrm{l})$ & $23.620 \pm 5.210$ & $4.727 \pm 1.321$ \\
Weight $(\mathrm{g})$ & $251.147 \pm 7.536$ & $323.133 \pm 13.192$ \\
Urine volume $(\mathrm{ml})$ & $91.100 \pm 9.463$ & $22.247 \pm 4.1028$ \\
Bladder wet weight $(\mathrm{mg})$ & $172.940 \pm 6.385$ & $135.833 \pm 2.302$ \\
\hline
\end{tabular}

Table III. NGF mRNA and protein expression $(\mathrm{pg} / \mathrm{ml})$ in the control, diabetic and treatment groups.

\begin{tabular}{lccr}
\hline Group & $\mathrm{n}$ & mRNA & \multicolumn{1}{c}{ Protein } \\
\hline Control & 15 & $0.183 \pm 0.004$ & $113.837 \pm 3.044$ \\
Diabetic & 15 & $0.032 \pm 0.139$ & $70.057 \pm 1.953$ \\
Treatment & 15 & $0.130 \pm 0.165$ & $109.943 \pm 2.347$ \\
\hline
\end{tabular}

NGF, nerve growth factor.

including pCDNA-3.1, while the transfection efficiency associated with the use of adenovirus vectors was markedly higher. However, the use of adenovirus vectors may lead to activation of an immune response, which is associated with a number of disadvantages during in vivo assays (10). Lentiviruses, an adenovirus subfamily, are able to infect cells of the majority of organisms, regardless of whether the cell is currently undergoing mitosis. Following insertion into the host genome, viral genes are stably expressed. The high infection rate and concentration of the virus enable 
long-term expression in vivo, high levels of safety and reduce host immune responses in the body (11). These advantageous properties have led to the use of lentiviruses as a common gene transfection vector.

The present study was performed using a novel, simple and efficient PCR cloning method, namely the in-fusion technology of Clontech Laboratories, Inc. (Mountain View, CA, USA). In-fusion technology is a newly developed technology suitable for use in the directed cloning of PCR products into linearized vectors using recombinase. Use of this technology in research is increasing and has been used for a number of applications, including genetic mutation, long fragment cloning and multi-gene fusion (12). Using this lentiviral technology, Berrow et al (13) obtained $>90 \%$ cloning efficiency during high-throughput vector construction, which is markedly higher than methods utilizing classic restriction ligases (14). In the current study, 15 bases, which were isogenous with the lentiviral vector, were connected to the ends of the NGF gene to construct the plasmid. Correct construction of the plasmid using the in-fusion technique was determined by PCR and sequencing.

Bone marrow MSCs were first described in 1968 by Friedenstein et al (15). MSCs are associated with a number of advantages, including numerous sources, easy separation and recovery, efficient amplification in vitro, no $\mathrm{MHC}$ II receptor expression and resistance to receptors $(16,17)$. MSCs differentiate into a wide diversity of cells under suitable conditions and are easily extracted autologously, avoiding immune rejection responses. These advantages have led to the development of MSCs into an important research tool (18-20). MSCs are easy to transfect and the transfected gene does not generally affect MSC differentiation and proliferation. Therefore, future applications of MSCs also include tissue and cell engineering and gene therapy. In the present study, rat MSCs were infected with a lentivirus transfected with a vector plasmid encoding a GFP tag or GFP tag and NGF. Infection efficiency and stable gene expression were confirmed by fluorescence microscopy. In addition, the exogenous NGF gene was identified to have been transcribed efficiently in rat MSCs and real-time PCR demonstrated that NGF expression was significantly greater in the NGF-transfected group, consistent with ELISA results, indicating that the MSCs secreted functional proteins.

For in vivo testing, rat MSCs expressing the therapeutic gene, NGF, were transplanted into diabetic rat bladders. Transplanted MSCs were revealed to exhibit markedly higher NGF expression in bladders of the treatment rats compared with the diabetic group. NGF gene expression in the bladder was stable and functional for use in diabetes therapy. This treatment is effective and safe as it was performed using autologous stem cells to prevent immune rejection responses and risks of viral infection, including increased active virus copies, activation of carcinogenic genes and insertion of mutations into the host genome. Further studies are required to investigate the differentiation of transplanted rat MSCs in the bladder of diabetic rats and determine variations in organizational structure and functions of the bladder following transplantation.

\section{References}

1. Ueda T, Yoshimura N and Yoshida O: Diabetic cystopathy: relationship to autonomic neuropathy detected by sympathetic skin response. J Urol 157: 580-584, 1997.

2. Paro M, Italiano G, Travagli RA, et al: Cystometric changes in alloxan diabetic rats: evidence for functional and structural correlates of diabetic autonomic neuropathy. J Auton Nerv Syst 30: 1-11, 1990.

3. Hellweg R, Raivich G, Hartung HD, Hock C and Kreutzberg GW: Axonal transport of endogenous nerve growth factor (NGF) and NGF receptor in experimental diabetic neuropathy. Exp Neurol 130: 24-30, 1994.

4. Sasaki K, Chancellor MB, Phelan MW, et al: Diabetic cystopathy correlates with a long-term decrease in nerve growth factor levels in the bladder and lumbosacral dorsal root ganglia. J Urol 168: 1259-1264, 2002.

5. Goins WF, Yoshimura N, Phelan MW, et al: Herpes simplex virus mediated nerve growth factor expression in bladder and afferent neurons: potential treatment for diabetic bladder dysfunction. J Urol 165: 1748-1754, 2001.

6. Sasaki K, Chancellor MB, Goins WF, et al: Gene therapy using replication-defective herpes simplex virus vectors expressing nerve growth factor in a rat model of diabetic cystopathy. Diabetes 53: 2723-2730, 2004.

7. Yokoyama T, Yoshimura N, Dhir R, et al: Persistence and survival of autologous muscle derived cells versus bovine collagen as potential treatment of stress urinary incontinence. J Urol 165: 271-276, 2001.

8. Strasser H, Marksteiner R, Margreiter E, et al: Transurethral ultrasonography-guided injection of adult autologous stem cells versus transurethral endoscopic injection of collagen in treatment of urinary incontinence. World J Urol 25: 385-392, 2007.

9. Huard J, Yokoyama T, Pruchnic R, et al: Muscle-derived cell-mediated ex vivo gene therapy for urological dysfunction. Gene Ther 9: 1617-1626, 2002.

10. Pedersini R, Vattemi E and Claudio PP: Adenoviral gene therapy in high-grade malignant glioma. Drug News Perspect 23: 368-379, 2010.

11. Dreyer JL: Lentiviral vector-mediated gene transfer and rna silencing technology in neuronal dysfunctions. Mol Biotechnol 47: 169-187, 2011.

12. Benoit RM, Wilhelm RN, Scherer-Becker D and Ostermeier C: An improved method for fast, robust, and seamless integration of DNA fragments into multiple plasmids. Protein Expr Purif 45: 66-71, 2006.

13. Berrow NS, Alderton D, Sainsbury S, et al: A versatile ligation-independent cloning method suitable for high-throughput expression screening applications. Nucleic Acids Res 35: e45, 2007.

14. Klock HE, White A, Koesema E and Lesley SA: Methods and results for semi-automated cloning using integrated robotics. J Struct Funct Genomics 6: 89-94, 2005.

15. Friedenstein AJ, Petrakova KV, Kurolesova AI and Frolova GP: Heterotopic of bone marrow. Analysis of precursor cells for osteogenic and hematopoietic tissues. Transplantation 6: 230-247, 1968.

16. Porada CD, Zanjani ED and Almeida-Porad G: Adult mesenchymal stem cells: a pluripotent population with multiple applications. Curr Stem Cell Res Ther 1: 365-369, 2006.

17. Hristov M, Heussen N, Schober A and Weber C: Intracoronary infusion of autologous bone marrow cells and left ventricular function after acute myocardial infarction: a meta-analysis. J Cell Mol Med 10: 727-733, 2006.

18. Kanazawa H, Fujimoto Y, Teratani T, et al: Bone marrow-derived mesenchymal stem cells ameliorate hepatic ischemia reperfusion injury in a rat model. PLoS One 6: e19195, 2011.

19. Choi H, Lee RH, Bazhanov N, Oh JY and Prockop DJ: Anti-inflammatory protein TSG-6 secreted by activated MSCs attenuates zymosan-induced mouse peritonitis by decreasing TLR2/NF- $\mathrm{KB}$ signaling in resident macrophages. Blood 118: 330-338, 2011.

20. Xu YL, Liu YL, Wang Q, Li G, Lü XD and Kong B: Intravenous transplantation of mesenchymal stem cells attenuates oleic acid induced acute lung injury in rats. Chin Med J (Engl) 125: 2012-2018, 2012. 